\title{
Estudio geológico y geofísico de la ladera sur del corte del mirador hidalgo en Ciudad Juárez, Chihuahua
}

\author{
Geological and Geophysical Study of the Hidalgo's Scenic \\ View South Hillside in Ciudad Juarez, Chihuahua
}

\author{
Dena-Ornelas O.S. \\ Centro de Información Geográfica \\ Instituto de Ingeniería y Tecnología \\ Universidad Autónoma de Ciudad Juárez \\ E-mail:oornelas@uacj.mx \\ Obeso-Cortez G. \\ Centro de Información Geográfica \\ Instituto de Ingeniería y Tecnología \\ Universidad Autónoma de Ciudad Juárez \\ E-mail:gocortez@gmail.com \\ Leyva J. \\ Centro de Información Geográfica \\ Instituto de Ingeniería y Tecnología \\ Universidad Autónoma de Ciudad Juárez \\ E-mail: jesusleyva@gmail.com
}

\author{
Domínguez-Acosta M. \\ Centro de Información Geográfica \\ Instituto de Ingeniería y Tecnología \\ Universidad Autónoma de Ciudad Juárez \\ E-mail:midoming@uacj.mx \\ Hernández-Jacobo V. \\ Departamento de Ingeniería Civily \\ Ambiental \\ Instituto de Ingeniería y Tecnología \\ Universidad Autónoma de Ciudad Juárez \\ E-mail:vihernan@uacj.mx
}

Granados-Olivas A.

Departamento de Ingeniería Civil y Ambiental

Instituto de Ingeniería y Tecnología

Universidad Autónoma de Ciudad Juárez

E-mail:agranados@uacj.com

De la Cruz-Cháidez S.T.

Departamento de Ingeniería Civil y Ambiental

Instituto de Ingeniería y Tecnología

Universidad Autónoma de Ciudad Juárez E-mail:schaidez@uacj.mx

Información del artículo: recibido: septiembre de 2009, aceptado: enero de 2011

\section{Resumen}

Los riesgos geológicos están asociados a procesos que reflejan el carácter dinámico del medio natural. La mayoría de las veces estos procesos han alcanzado un punto de equilibrio o estabilidad. Sin embargo, en ocasiones la actividad humana interfiere en la naturaleza, modificando las condiciones existentes de equilibrio de los macizos rocosos, liberando de forma súbita enormes fuerzas asociadas a la energía potencial acumulada durante millones de años. En este estudio se analiza el efecto antrópico de desequilibrio inducido sobre la Sierra de Juárez como consecuencia de uno de los cortes (mirador hidalgo) practicado sobre la misma para dar paso a obras de infraestructura vial. El enfoque aplicado consistió en integrar mediante un Sistema de Información Geográfica (SIG), mapas topográficos de alta resolución, análisis de terreno, mapeo geológico, tomografías eléctricas resistivas (TER) y sondeos electromagnéticos en el dominio del tiempo (TEM) para dimensionar el grado de afectación que el Mirador Hidalgo representa en términos de un Índice de Susceptibilidad Geológica (ISG). A partir de los resultados obtenidos se propone una serie de acciones inmediatas que deben considerarse para evitar que esta obra cause daños a la infraestructura y/o pérdida de vidas humanas.

\section{Descriptores}

- riesgo geológico

- Sierra de Juárez

- Índice de Susceptibilidad Geológica (ISG)

- Tomografías Eléctricas Resistivas (TER)

- Sondeos Electromagnéticos en el Dominio del Tiempo (TDEM) 


\begin{abstract}
Geologic risks are associated to the different processes inherited by the dynamic condition of the natural environment. Often, these processes have reached a natural state of equilibrium or stability. Nevertheless, in some instances human activity interferes with the natural order, modifying the existing equilibrium or balance state. Such is the example of some geologic rock outcrops, originally generated under pressure and temperature conditions, different from the ones found at the surface of the earth. These outcrops, when subjected to surface atmospheric conditions experience a sudden release of large forces associated to the potential energy accumulated over long periods of time (thousands to millions of years). The goal of this study is to focus on the anthropogenic disequilibrium induced over a segment of the Sierra de Juarez, consequence of the removal of large rock masses in the construction of a scenic point (Mirador Hidalgo) and a traversing highway (Camino Real). The applied methodology consisted in the integration through a Geographic Information System (GIS) of high-resolution topographic maps, terrain analysis, geologic mapping, electric resistivity tomography and time domain electromagnetic soundings as a mean to evaluate the potential hazard that the Mirador Hidalgo represents in terms of an arbitrary Geologic Susceptibility Index (GSI). Based on the obtained results, a series of immediate actions are proposed, which are to be considered in order to avoid continuing damage to the highway infrastructure as well as the potential loss of human lives.
\end{abstract}

\section{Introducción}

Los fenómenos naturales asociados a los riesgos geológicos son, entre otros: procesos de erosión, procesos de disolución, movimientos sísmicos, erupciones volcánicas e incluso precipitaciones pluviales atípicas o extraordinarias que induzcan deslizamientos y desprendimientos de tierra en taludes y laderas, así como derrubios y subsidencias del terreno. Los riesgos geológicos se cuantifican en términos de susceptibilidad, probabilidad de ocurrencia o peligrosidad y la estimación de vulnerabilidad y costos (González et al., 2002; Ayala, 1987; Mendoza et al., 2002). El grado de susceptibilidad y peligrosidad que presenta el terreno se determina con base en un inventario de factores condicionantes y desencadenantes. Los primeros son la descripción en sí del medio geológico como tal, en tanto que los desencadenantes son los elementos, tanto naturales como antropogénicos, que actúan como mecanismo de disparo para que un evento sea considerado como desastre geológico. La conjunción de estos factores, en el caso de la construcción de la obra de infraestructura vial conocida como Camino Real, localizada geográficamente al noreste de las faldas de la Sierra de Juárez, en la periferia de la fronteriza Ciudad Juárez, al norte del estado de Chihuahua, en México (figura 1), significó una alteración importante del escenario geológico, al practicarse una serie de cortes en los macizos rocosos localizados a lo largo del trazo de esta obra.
El corte del mirador hidalgo, una de las tantas remociones de masa rocosa realizadas durante la construcción de la vialidad Camino Real, está practicado sobre un terreno originalmente considerado como compuesto por conglomerados Terciarios, conforme a los mapas geológicos publicados (Drewes y Dyer, 1993). Estos materiales, asociados a rellenos de tipo coluvial depositados sobre las estribaciones de los macizos rocosos de caliza que conforman la Sierra de Juárez, se consideraban estables. Sin embargo, una vez realizada la obra se advirtió que el escenario geológico era mucho más complejo de lo esperado, presentando una serie de factores condicionantes adversos para la edificación de obra civil, tales como: alteraciones de contacto, presencia de intrusivos, fallas geológicas (Ávila, 2005; Keaton, 1993; Lovejoy, 1976; Raney y Collins, 1990) fracturamientos y discontinuidades extensas, así como litologías débiles (Drewes y Dyer, 1993; Rascón y Gómez, 2008; Dena, 2009).

Esta problemática se agudizó ante el efecto de los procesos de meteorización que han ocasionado que una cantidad importante de derrumbes o detritos de roca invadan el primer carril de la vialidad Camino Real.

El objetivo de la presente investigación es el de evaluar, en términos de un Índice de Susceptibilidad Geológica (ISG), las condiciones actuales del macizo rocoso que soportan la infraestructura del mirador hidalgo (figura 1). 


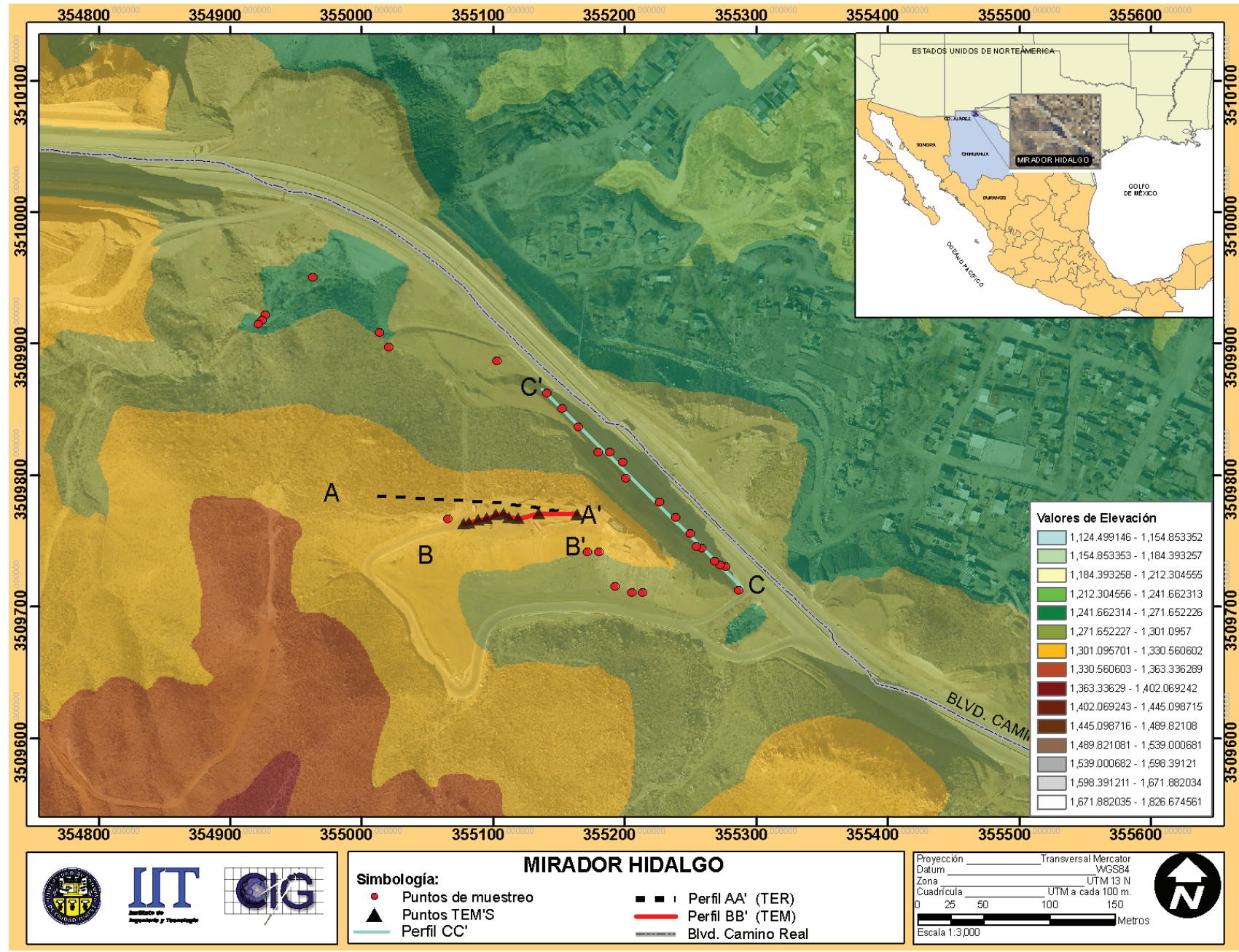

Figura 1. Mapa de localización del área de estudio, donde se encuentra el mirador hidalgo

\section{Descripción geológica general}

La Sierra de Juárez está localizada al oeste de la mancha urbana de Ciudad Juárez (figura 1) y está conformada por rocas sedimentarias de tipo marino depositadas durante el periodo Cretácico (65-144 Ma) (Drewes y Dyer, 1993). Estas unidades sedimentarias muestran intensos patrones de deformación tales como plegamientos y fallas de tipo compresional y fábricas dominantes de grano orientado al noroeste (Drewes y Dyer, 1993). La Sierra de Juárez se separa estructuralmente por el lineamiento de Texas de las formaciones de edad Paleozoica y Precámbrica no deformadas internamente, que conforman las capas basculadas de las Montañas Franklin (Haengii, 2002). El escenario geológico es consecuencia de procesos tectónicos en los que un régimen de esfuerzos compresionales deformó las unidades sedimentarias durante el Paleoceno (55-65 Ma) (Drewes y
Dyer 1993). Posteriormente, durante el periodo Eoceno medio (ca. $40 \mathrm{Ma}$ ), las rocas sedimentarias y algunas fallas fueron invadidas de forma transgresiva y concordante por cuerpos intrusivos (diques y silletas) (Drewes y Dyer, 1993). Finalmente, cuando la actividad ígnea cesó, la activación de fallas extensionales asociadas al sistema fisiográfico de cuencas y sierras ( 20 Ma) ocasionó la topografía resultante (Drewes y Dyer, 1993; Haengii, 2002). Tectónicamente, la Sierra de Juárez está emplazada en la provincia del sistema sísmicamente activo de apertura continental del Río Grande Rift (Keller et al., 1990; Haller et al., 2002), el cual controla estructuralmente la zona a través de un sistema de fallas normales recientes (cuaternarias, $1.8 \mathrm{Ma}$ ) (Drewes y Dyer, 1993; Collins y Raney, 1991; Machette, 1987; Keaton y Barnes, 1996; Haller et al., 2002).

Litoestratigráficamente, las unidades sedimentarias presentes en la Sierra de Juárez son tanto rocas calizas 
de estratificación gruesa, catalogadas como de tipo masivo (Formación Benigno, Lágrima y Finlay) que representan unidades estables para la cimentación y anclaje de estructuras (Flores et al., 2001), como rocas calizas de estratificación delgada, y lutitas proclives al proceso de disolución (Formación Cuchillo y Del Río), así como unidades sedimentarias de tipo granular como conglomerados y arcillas (Drewes y Dyer, 1993). Los planos de estratificación entre unidades estables (calizas de estratificación gruesa) e inestables (calizas y lutitas de estratificación delgada y plegadas) se consideran como zonas que han sido debilitadas como consecuencia de los procesos tectónicos (Drewes y Dyer, 1993), presencia de intrusivos (Drewes y Dyer, 1993) e intemperismo por actividad meteórica; en tanto que las unidades sedimentarias granulares de edad reciente (arcillas, conglomerados y rellenos de tipo aluvial), sólo han sido afectadas por procesos hidrometeorológicos (CNA, 2008; Rascón y Gómez, 2008; Dena, 2009).

\section{Metodología}

La metodología aplicada en este estudio es una modificación a la propuesta por González de Vallejo et al. (2002) (figura 2), en la cual se definen y describen diferentes niveles de detalle en estudios enfocados a evaluar el riesgo asociado con procesos geológicos. En este estudio en particular, el alcance se limitó a describir, de forma cualitativa, una sección o perfil de la ladera sur del mirador hidalgo con base en un ISG, en el que además de los estudios a nivel de mapa de inventario y factores condicionantes, se incorporaron factores des-

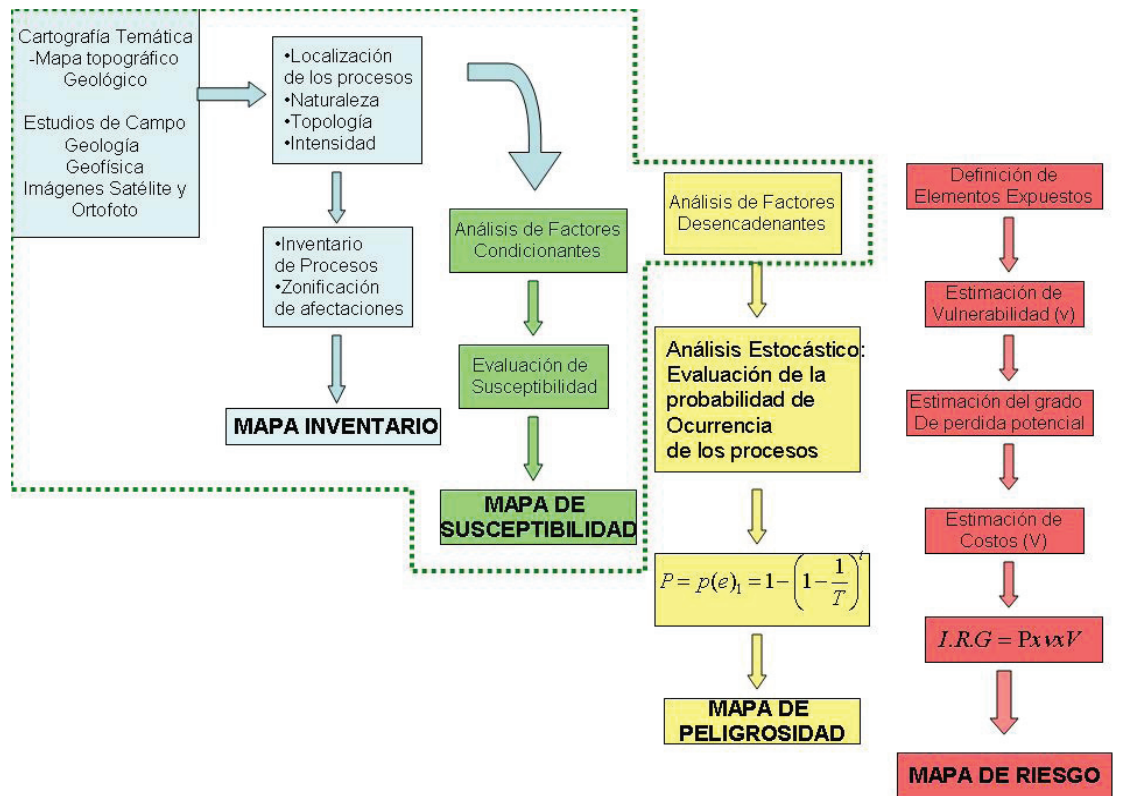

encadenantes (polígono punteado verde (figura 2)), sin llegar al nivel de modelación estocástica que involucraría un análisis de peligrosidad geológica. En una primera etapa se estudiaron mapas geológicos existentes del área de estudio (Drewes y Dyer, 1993; Rascón y Gómez, 2008), seguido por la evaluación de imágenes en plataforma QuickBird (CIG, 2005) y ortofoto obtenida con resolución de $1 \mathrm{~m} \times 1 \mathrm{~m}$ adquirida mediante vuelo LIDAR (Light Data Acquisition Altitude and Range) (IMIP, 2007), para identificar las variaciones de carácter geomorfológico practicadas sobre el terreno. Posteriormente, se realizaron una serie de estudios de campo consistentes en caminamientos geológicos para la identificación tanto de unidades litoestratigráficas, como de elementos estructurales tales como fallas, familias de discontinuidades, planos de contacto y demás elementos requeridos para describir un macizo rocoso (González et al., 2002).

La continuidad tridimensional de los elementos mapeados en superficie, expuestos e identificados en el corte, se infirió a través de estudios geofísicos que combinaron métodos eléctricos y electromagnéticos. De entre los métodos eléctricos, se seleccionó la técnica de la Tomografía Eléctrica Resistiva (TER), la cual consiste en generar secciones o perfiles del subsuelo con base en mediciones de caída de potencial eléctrico (Dahlin y Zhou, 2004; Loke, 2000). El principio físico es esencialmente el mismo que el de los Sondeos Eléctricos Verticales (SEVs), es decir, se inyecta una corriente directa mediante dos electrodos de corriente y se mide la diferencia o caída de potencial asociada a la resistividad del subsuelo por medio de dos electrodos de potencial, conectados a un voltímetro de alta impedancia (Sharma, 1997). La variación que introduce la técnica de la TER respecto de los SEVs, consiste en que de forma más expedita se lleva a cabo una consecución lineal de sondeos eléctricos verticales, variando el espaciamiento entre electrodos con el objetivo de alcanzar mayores profundidades de exploración (figura 3). Al igual que en los SEVs, existen diversos arreglos electródicos, seleccionándose para este estudio el arreglo Wenner-Alpha descrito por Loke $(2000,2004)$.

Figura 2. Definición de inventario, susceptibilidad, peligrosidad y riesgo geológico (modificado de González de Vallejo et al., 2002) 


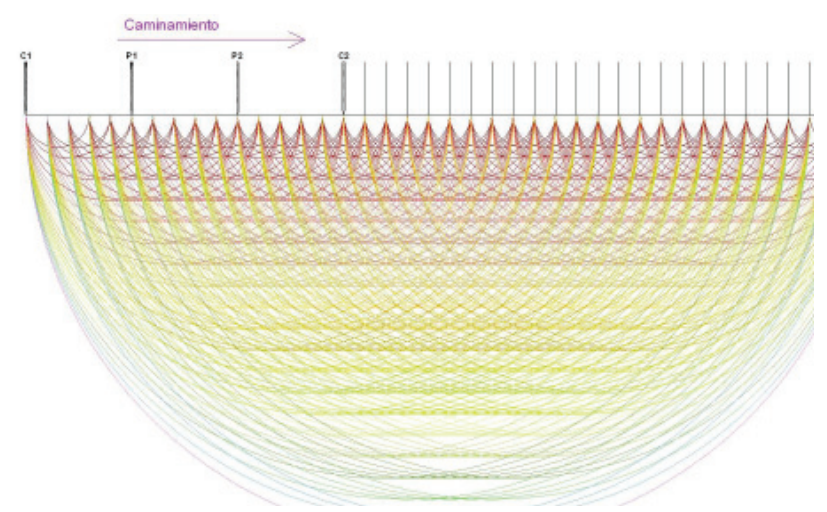

Figura 3. Disposición de los electrodos en superficie para realizar una tomografía eléctrica resistiva

En cuanto a métodos electromagnéticos, en este estudio se aplicó la técnica de los Sondeos Electromagnéticos en el Dominio del Tiempo (TDEM), la cual, igual que los métodos eléctricos, mide variaciones de resistividad de las capas que conforman el subsuelo (Shah et al., 2005), pero a diferencia de los SEVs, no utiliza pulsos rectangulares de corriente directa, sino que usa corriente alterna circulando a través de un conductor en forma de espiral en superficie, para generar un campo magnético primario que induce una corriente en el subsuelo (Telford, et al., 1995), la cual a su vez, genera un campo magnético secundario que es medido por una bobina de recepción en superficie (figura 4), determinándose la capacidad de conducción eléctrica del subsuelo o su recíproco, la resistividad, permitiendo de esta manera discriminar las unidades litológicas, dado que los diferentes materiales que las conforman presentarán diferentes resistividades (Palacky, 1987).

Finalmente los ISG se generaron mediante intersecciones de las variables que definen los factores condicionantes y desencadenantes. Para este efecto se generó una matriz que permitiera parametrizar o cuantificar el peligro por procesos geológicos mediante la asignación de valores a los factores condicionantes y desencadenantes identificados en el corte. Se asignaron valores entre 0 y 1 , al mínimo y máximo de susceptibilidad geológica, respectivamente. El valor final se calculó mediante la sumatoria de los valores asignados a cada proceso del inventario, dividido por el total de procesos considerados.

\section{Datos y procesamiento}

Los datos geofísicos de la TER fueron recabados utilizando un equipo ABEM Terrameter SAS4000 (ABEM, 2008), mediante un solo tendido de 75 electrodos, distribuidos a lo largo de $160 \mathrm{~m}$ de cable, localizado sobre

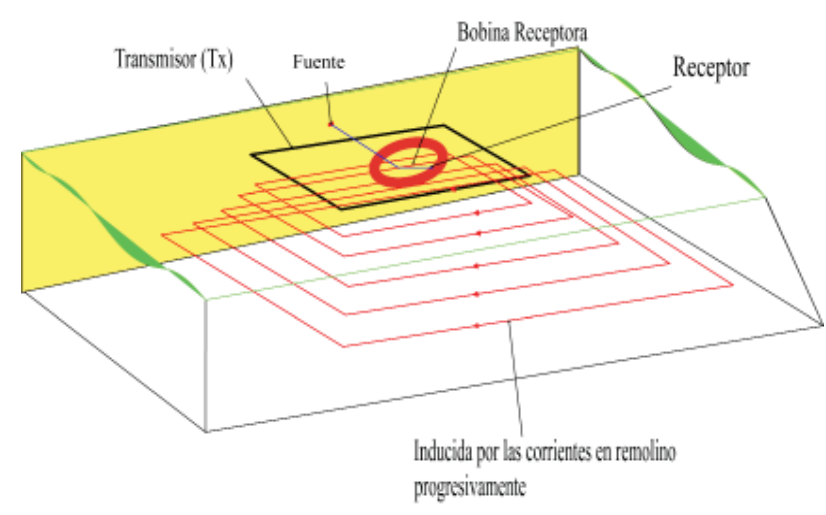

Figura 4. Arreglo del sondeo electromagnético en el dominio del tiempo (modificado de Shah et al., 2005)

la parte superior del mirador hidalgo hasta donde la geometría del corte y las condiciones de seguridad lo permitieron (figura 1). El equipo fue programado para adquirir dos secuencias diferentes del arreglo tipo Wenner-Alpha (Loke, 2000, 2004): en la primera secuencia se utilizó una separación de electrodos de $4 \mathrm{~m}$ para alcanzar profundidades del orden de los $25 \mathrm{~m}$, en tanto que en la segunda secuencia se inyectó la corriente con una separación de $2 \mathrm{~m}$ entre electrodos para densificar la información recabada en la sección media del tendido con una mayor resolución espacial.

Las resistencias recabadas en campo fueron corregidas geométricamente mediante el paquete computacional ERICGRAPH@ (Dahlin y Liderman 2007), para posteriormente convertir los valores de resistividad aparente mediante el algoritmo de inversión robusta del programa RES2DINV (Geotomo Software, 2003) a resistividad verdadera. La sección geoeléctrica (perfil $\mathrm{BB}^{\prime}$ ) (figura 5) resultante registró un error RMS de $8.2 \%$, con un $85 \%$ de los valores de resistividad aparente obtenidos con un error menor al 10\%. En cuanto a los estudios electromagnéticos, se utilizó un equipo PROTEM 47 de Geonics Limited (1999) para realizar 10 TEMs, distribuidos casi de forma coincidente con la TER (figura 1, triángulos negros), pero alcanzando mayores profundidades de penetración (100 m, aproximadamente). El arreglo utilizado consistió en colocar una bobina de recepción $(\mathrm{Rx})$ de $1 \mathrm{~m}$ de diámetro al centro de una bobina de trasmisión (Tx) con una longitud de cara de $10 \mathrm{~m}$ (figura 4), ambas bobinas siempre estuvieron colocadas de forma horizontal sobre la superficie plana de la carpeta del estacionamiento para evitar distorsiones del campo magnético primario. La intensidad de corriente osciló en un rango comprendido entre 2.5 y 3 amperes, y los datos se adquirieron en tres frecuencias: $285 \mathrm{~Hz}, 75 \mathrm{~Hz}$ y $30 \mathrm{~Hz}$. Los tiempos de apagado y encendido para la sincronización entre el Rx y el Tx se fijaron con duración 
de $0.8 \mu$ s. Las mediciones se realizaron con ganancias diversas, aumentando el número de lecturas en sitios donde el ruido geológico era mayor, esto con el fin de aumentar la calidad en la estadística de los datos. Los datos resultantes se modelaron en 1D mediante el algoritmo de inversión de OCAMM (Constable et al., 1987). Finalmente, se generó una rejilla o arreglo matricial interpolado ("grid") usando el paquete computacional Generic Mapping Tools (Wessel y Smith), para determinar las correlaciones geoespaciales entre los sondeos invertidos 1D, facilitando así su interpretación. En esta sección es importante aclarar que los modelos resultantes son intrínsecamente limitados dado el hecho de que los procesos de inversión 2D de la TER y $1 \mathrm{D}$ de los TEMs, asumen que la resistividad no varía perpendicularmente al perfil y continuidad horizontal de capas, respectivamente.

\section{Resultados}

Los dominios resistivos de los modelos invertidos tanto de TEMs como de la TER se clasificaron en Unidades Geoeléctricas (UGE), asociando sus rangos resistivos a litologías probables con base en valores y tabulaciones publicadas (Palacky, 1987; Sharma, 1997), así como a resultados de estudios anteriores publicados (CNA, 2008) y no publicados (Dena, 2009). La clasificación resultante (tabla 1) muestra las litologías inferidas geofísicamente.

La UGE1 se subdivide en dos subdominios resistivos: la subunidad UGE1a presenta resistividades comprendidas en el rango de $30-40 \Omega-\mathrm{m}$, valores posiblemente asociados con litologías compuestas por arenas limosas, en tanto que la subunidad UGE1b muestra valores resistivos comprendidos entre los 40-160 $\Omega-\mathrm{m}$, asociados a conglomerados de edad terciaria, compuestos por arenas limosas interestratificadas por capas de boleos de caliza de diámetro menor a $10 \mathrm{~cm}$. Las subunidades UGE2a y UGE2b componen el dominio resistivo UGE2, que presenta los valores mayores de resistividades registrados: Rho $\geq 300 \Omega$-m; tales va-

Tabla 1. Clasificación de unidades geoeléctricas

\begin{tabular}{c|c|c|l}
\hline UGE & Sub- UGE & Resistividad & \multicolumn{1}{c}{ Litología } \\
\hline \multirow{2}{*}{ UGE1 } & UGE1a & $30-40 \Omega-\mathrm{m}$ & Arenas limosas \\
& UGE1b & $40-160 \Omega-\mathrm{m}$ & $\begin{array}{l}\text { Conglomerado terciario dominado por arena } \\
\text { limosa } \\
\end{array}$ \\
UGE2a & $>300 \Omega-\mathrm{m}$ & Conglomerado cementado \\
UGE2 & UGE2b & $>300 \Omega-\mathrm{m}$ & Rocas ígneas (intrusivo) o calizas \\
UGE3 & & $10-80 \Omega-\mathrm{m}$ & Contacto entre rocas ígneas (ó calizas) con lutitas \\
UGE4 & & $<8 \Omega-\mathrm{m}$ & Lutitas \\
\hline
\end{tabular}

lores de resistividad están asociados, en el caso de la subunidad UGE2a, a un paquete de conglomerado cementado compuesto por boleos de caliza; en tanto que la litología asignada a la subunidad UGE2b es roca ígnea de tipo andesítico o caliza perteneciente al periodo Cretácico (Drewes y Dyer, 1993; Rascón y Gómez, 2008; Dena, 2009). La unidad UGE3, con valores resistivos en el rango de 10-80 $\Omega-\mathrm{m}$, se consideró en este estudio como una posible zona de contacto (ZC) o transición entre rocas sedimentarias (calizas) o intrusivas ígneas (lutitas). Dependiendo de consideraciones geométricas o de estructura, el contacto puede ser interpretado como una zona de falla. Finalmente, la UGE4, con resistividades menores a los $8 \Omega$-m, está asociada con lutitas.

En cuanto a la distribución geoespacial de las UGEs, en el perfil A-A' (figura 5) se observa que la geometría del dominio UGE2a muestra un espesor de $4 \mathrm{~m}$ en el extremo oeste del perfil, adelgazándose hasta $1 \mathrm{~m}$ en la sección media, siendo interrumpida por las subunidades UGE1a y UGE1b, que impiden la continuidad lateral de la UGE2a, la cual aparece nuevamente hacia el este del perfil con un espesor de $4 \mathrm{~m}$. En cuanto a la subunidad UGE1a, de un espesor promedio de $7.5 \mathrm{~m}$ aproximadamente en el extremo oeste del perfil, se adelgaza hasta 1 $\mathrm{m}$, aflora hacia la superficie a partir de la coordenada de modelo (C.M.) $60 \mathrm{~m}$, y desaparece a los $120 \mathrm{~m}$ en C.M. La subunidad UGE1b subyace a la UGE1a a partir de la C.M. $60 \mathrm{~m}$ con un espesor de $2.5 \mathrm{~m}$, aumentando hasta $7.5 \mathrm{~m}$ en los $106 \mathrm{~m}$ C.M., y termina con un grosor de $5 \mathrm{~m}$, sobreyaciendo la UGE2a, en el este del perfil. La subunidad UGE2b se ve interrumpida en su continuidad por una zona de contacto (ZC), entre las C.M. 60 y 70 m, desapareciendo a los $116 \mathrm{~m}$ C.M. La UGE3 muestra valores resistivos en el rango de 10-80 $\Omega-\mathrm{m}$, a los 116 m en C.M., como consecuencia de la posible presencia de zonas de falla con cuñas verticales de lutita observadas en los contactos entre rocas de alta resistividad (calizas y/o ígneas). Finalmente, la UGE4 se asocia a paquetes de lutita con ángulos de echado mayores a los $70^{\circ}$ embebidos o interestratificados, entre paquetes de rocas ígneas y/o calizas. Estos patrones estructurales y de estratificación han sido reportados en estudios geofísicos y recorridos geológicos previos (Rascón y Gómez, 2008; Dena, 2009). 


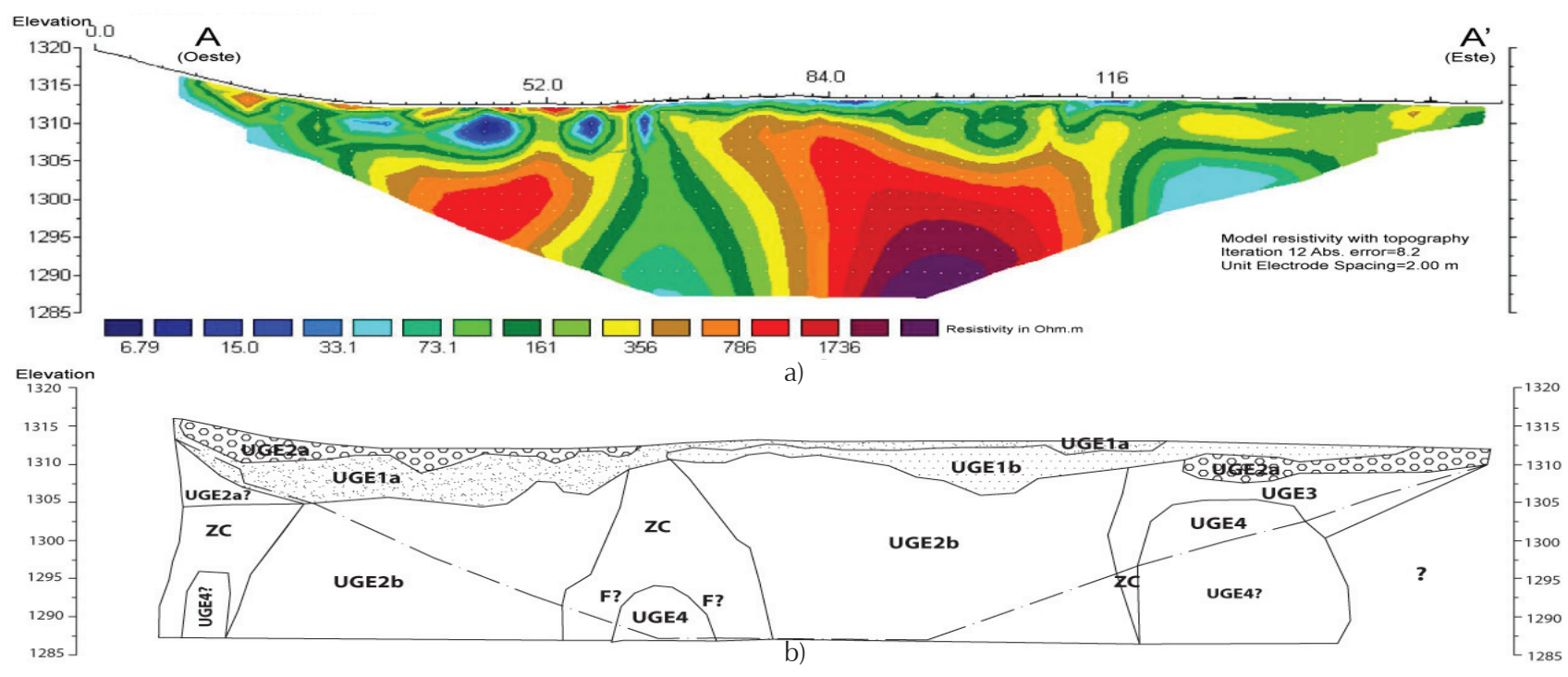

Figura 5. a) Sección resistiva $A A^{\prime}$, b) Modelo litoestratigráfico interpretado; la línea punteada representa el limite de la TER

En el perfil B-B' (figura 6), correspondiente al modelo interpolado, invertido en 1D, de los sondeos TEM, se observa que la geometría de la subunidad UGE1b es prácticamente uniforme, mostrando un espesor aproximado de $15 \mathrm{~m}$ al extremo oeste del perfil, engrosándose hasta aproximadamente $20 \mathrm{~m}$ en la sección media; finalmente, se estrecha con un espesor de $7 \mathrm{~m}$ en el extremo este del perfil, donde se acuña entre los limites de los dominios resistivos UGE1a y UGE2b. La subunidad UGE1a es apenas apreciable a partir de la localización del TEM 9, engrosándose en dirección este hasta alcanzar un espesor máximo de $2 \mathrm{~m}$ a la altura del TEM 10. La UGE2b muestra su máximo espesor, aproximadamente $25 \mathrm{~m}$, en el extremo oeste del perfil, desvaneciéndose prácticamente hasta la altura de la sección media; los valores resistivos altos se observan nuevamente a partir del TEM 9, acuñándose hasta alcanzar un espesor de $10 \mathrm{~m}$, aproximadamente, en el extremo este del perfil, en la vecindad del TEM 10. Finalmente, las dos subunidades UGE3 y UGE4, muestran un comportamiento geométrico no uniforme, en el cual la lutita se adelgaza en dirección este hasta el TEM 3 para engrosarse significativamente a partir del TEM 7, donde se observan los mínimos valores resistivos registrados. Esta compleja geometría es asociada a una zona de contacto entre litologías de tipo ígneo o caliza y lutita, así como la presencia de fallas y/o pliegues.

A pesar de las limitaciones de los procesos de inversión tanto en la TER como en los TEMs, se observó que había concordancia de los resultados del modelado con los elementos estructurales y litológicos identificados en el reconocimiento de campo, siendo entonces la in- terpretación anteriormente descrita consistente con los elementos estructurales presentes, tanto en las inmediaciones como en el corte del mirador hidalgo.

\section{Descripción del macizo rocoso}

Entre los factores condicionantes más sobresalientes identificados como resultado de los caminamientos geológicos realizados (perfil CC', figura 7), se observó que el patrón de fracturamiento es intenso en la parte sureste del corte, disminuyendo ligeramente después de 100 m en C.M. en dirección noroeste. En cuanto a elementos estructurales, la falla más importante (F1 en la figura 7), dada su caracterización geológica, es de tipo compresional con un desplazamiento apreciable en los estratos de lutita. Esta falla controla geológicamente el corte y define en consecuencia la zona más debilitada que se observa en el macizo rocoso expuesto en el talud. Es entre esta falla geológica y el extremo sureste del mirador, donde se observa el mayor grado de afectación del edificio geológico y la infraestructura sobreyaciente. En lo referente a contactos litológicos y estratigrafía, se identificaron cinco unidades litológicas representativas del corte (figuras 7 y 8): (1) dos familias de lutitas $(\mathrm{Lu})$, una de color sepia púrpura y otra de color negro mate, posiblemente asociado con un alto contenido de material orgánico, (2) rocas calizas de tipo masivo $(\mathrm{Cz})$, (3) intrusivos terciarios de composición andesítica (Ig), (4) conglomerado terciario $(\mathrm{Cg} \mathrm{Tc})$ conformado por materiales calcáreos y (5) rellenos antropogénicos (RA) formados por detritos de lutitas, calizas y fragmentos angulares de roca 

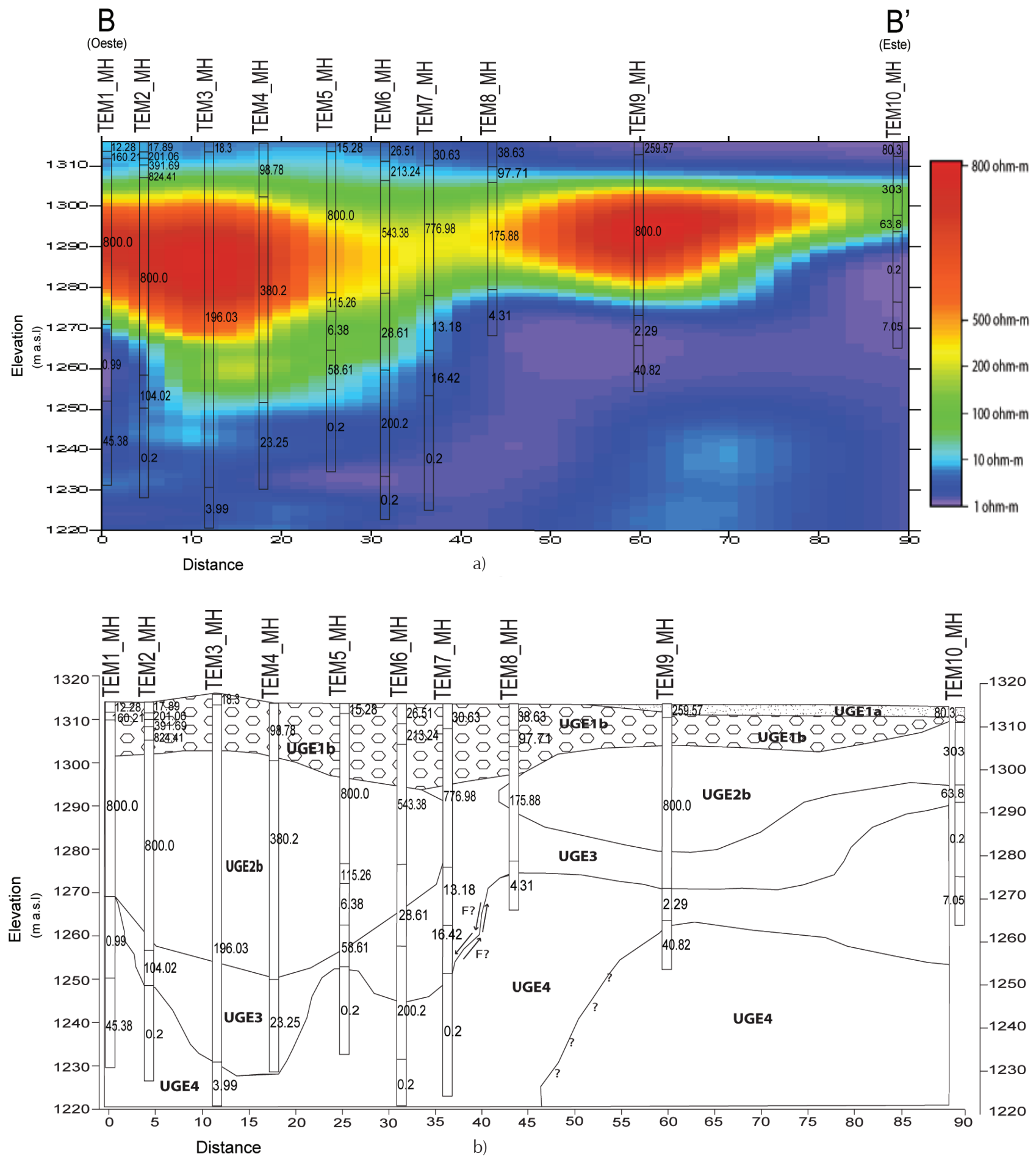

Figura 6. a) Sección resistiva BB' correspondiente a los sondeos TEM, b) Modelo litoestratigráfico interpretado 


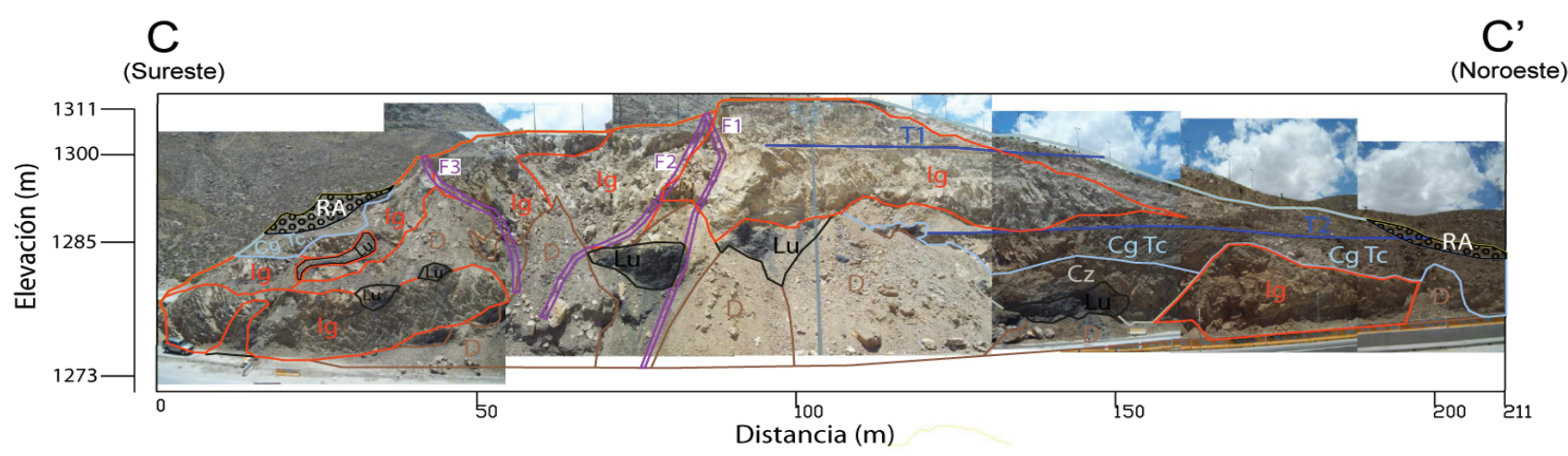

Figura 7. Interpretación de las unidades litológicas, estratigráficas, estructurales y geomorfológicas del corte del mirador hidalgo

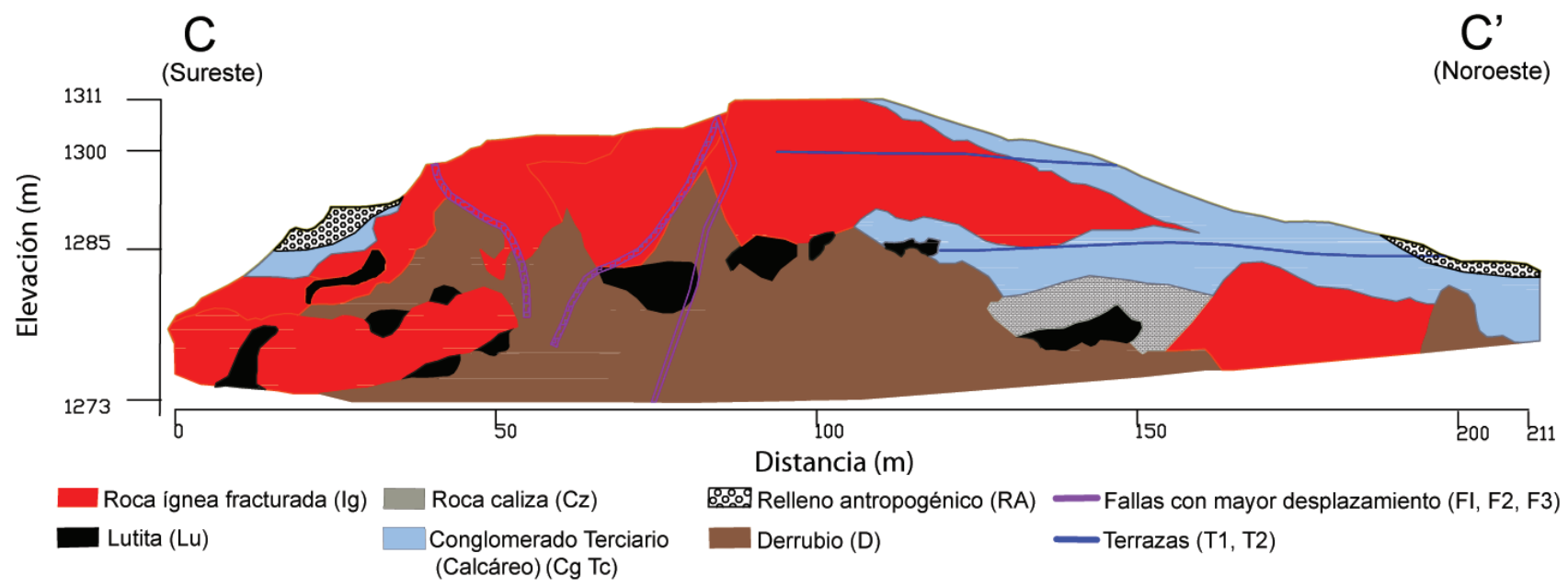

Figura 8. Perfil geológico interpretado del corte del mirador hidalgo

ígnea intrusiva, como consecuencia de las explosiones realizadas para practicar el corte sobre el macizo rocoso. No se detectó la presencia de mantos acuíferos en la zona. Respecto a las propiedades mecánicas, los valores de resistencia a la compresión simple de los materiales expuestos en los afloramientos de roca en el corte del mirador, se determinaron cualitativamente con base en los parámetros de la ISRM (1978), registrándose para cada litología los siguientes valores: $\mathrm{Lu}$ : $\mathrm{R} 0$, correspondiente a un rango de 0.25 a $1 \mathrm{MPa}$; $\mathrm{Cz}$ : R5, 100 a 250 Mpa; Ig: R4, 100 a 250 Mpa; Cg Tc: R1, 1 a 5 Mpa; y RA: R0, 0.25 a $1 \mathrm{Mpa}$.

En lo que respecta a factores desencadenantes, la ocurrencia extraordinaria de eventos hidrometeorológicos extremos es sin duda de los más importantes a considerar, dado que se han registrado tormentas de hasta $80 \mathrm{~mm}$ distribuidas en 24 horas (Laboratorio de Climatología de la UACJ, 2006), las cuales corresponden a un periodo de retorno de 20 años (CNA, 2008). De igual manera, las relativamente extremas bajas temperaturas durante los crudos inviernos septentrionales del estado de Chihuahua son un factor incidente en los procesos de intemperización meteórica de las rocas ígneas, principalmente al provocar una rápida contracción y expansión de dichas rocas. En cuanto a los posibles cambios en las condiciones hidrogeológicas, el efecto se consideró nulo, dado que no se observaron mantos freáticos o acuíferos colgantes en el interior del macizo. En lo referente a pérdidas de estabilidad por causas geométricas, se identificó un incremento de las cargas sobre la ladera, asociado a la construcción de obras y acumulación de materiales, además de cambios en la topografía e imposición de sobrecargas, debido al incremento del ángulo del talud durante la ejecución del corte. Además, se identificaron litologías susceptibles de cambiar sus propiedades de resistencia mecáni$\mathrm{ca}$, tales como conglomerados terciarios, lutitas e intrusivos ígneos.

En resumen, la interpretación de los resultados globales de los caminamientos geológicos y estudios geofísicos revelan que, en términos de estructura, el área de estudio presenta un intenso patrón de falla- 
miento, fracturamiento y plegamiento atribuido a eventos de carácter tectónico, presencia de intrusivos ígneos y actividad antrópicas que, en conjunto, han debilitado mecánica y estructuralmente el macizo rocoso del mirador hidalgo.

\section{Zonificación del índice de susceptibilidad geológica}

Para dimensionar la susceptibilidad de riesgo geológico en el área, se procedió a zonificar el corte (figura 8), en términos de las estructuras geológicas observadas como se describe a continuación:

Zona I: Región comprendida entre el extremo sureste y la falla F1.

Zona II: Región comprendida entre la falla F1 y la zona en la cual las terrazas de estabilización (T1 y T2) están integradas estructuralmente.

Zona III: Región comprendida entre la zona, en la cual las terrazas de estabilización (T1 y T2) están integradas estructuralmente y la zona de rocas calizas expuestas $(\mathrm{Cz})$.

Tabla 2. Determinación del ISG

\begin{tabular}{lcccc}
\hline \multicolumn{1}{c}{ Factores condicionantes } & Zona 1 & Zona ll & Zona lll & Zona IV \\
\hline Fallas & 1 & 0.7 & .3 & .25 \\
Litología y estratigrafía & 1 & 0.8 & .7 & .25 \\
Hidrogeología & 0 & 0 & 0 & 0 \\
Propiedades físicas resistivas & 1 & 0.8 & .4 & .2 \\
Factores desencadenantes & & & & \\
Sobrecargas estáticas & 1 & .9 & .7 & 0 \\
Cargas dinámicas & 0 & 0 & 0 & 0 \\
Cambios en las condiciones & 0 & 0 & 0 & 0 \\
hidrogeológicas & 1 & 1 & .75 & 0.5 \\
Factores climáticos & 1 & 1 & 0.5 & 0.25 \\
$\begin{array}{l}\text { Variaciones en la geometría } \\
\text { Reducción de parámetros }\end{array}$ & 0.8 & 0.3 & 0.25 & 0 \\
resistivos & 68 & 55 & 36 & 14.5 \\
Totales \% & & & & \\
\hline
\end{tabular}

Zona IV: Comprendida entre el inicio de las calizas expuestas y el extremo noroeste, conformado por conglomerado terciario ( $\mathrm{Cg} \mathrm{Tc})$.

Posteriormente, con base en la correlación de los factores condicionantes y desencadenantes con su distribución geoespacial, se asignaron valores o pesos a cada factor de la siguiente manera: el máximo de elementos a cada factor se normalizó como uno y cero a la ausencia total del mismo, es decir, por ejemplo, en la Zona I se observó el mayor numero de contactos, fallas y fracturas, entonces a ese total se le asignó arbitrariamente el factor de peso 1 o 100\% (tabla 2), en tanto que en la Zona IV donde sólo se observó un numero de contactos, fallas y fracturas que totalizaban aproximadamente la cuarta parte de lo indicado en la Zona I, se le asignó un peso de 0.25 o $25 \%$ (tabla 2). En los demás factores se procedió de forma similar, dándose el caso en que algunos factores, como el hidrogeológico, dada la nula presencia de mantos freáticos, no incidían en lo absoluto en la estabilidad del mirador hidalgo. En el caso del factor de reducción de parámetros resistivos se asignó un máximo de $80 \%$ (peso de 0.8 ) a la Zona I, ya que aunque la roca ígnea parece ser estable (Grado de resistencia R3, (ISRM, 1978)), le subyace en algunos casos, paquetes severamente alterados de lutita (Grado de resistencia R0 (ISRM, 1978)). Mediante este enfoque, finalmente se obtuvo un ISG, que si bien es arbitrario, no deja de ser representativo del riesgo geológico presente en el mirador (tabla 2).

Finalmente, los resultados de las sumatorias de la tabla 2 se representaron con polígonos codificados con diferentes sombreados (figura 9) para representar el ISG: los tonos 'fríos' se correlacionan con poco riesgo, en tanto que los tonos 'cálidos' se asocian con zonas de relativamente alta peligrosidad geológica.

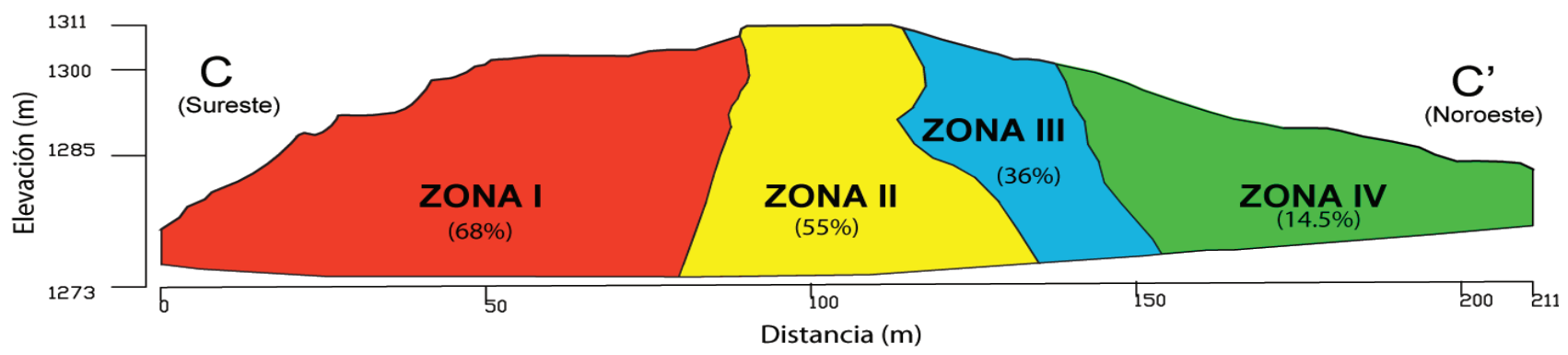

Figura 9. Perfil de zonificación de susceptibilidad geológica del corte del mirador hidalgo 


\section{Conclusiones}

La descripción del macizo rocoso se realizó con base en los elementos estructurales y litologías observadas tanto en las inmediaciones como en el talud del mirador en la fase de reconocimiento de campo. El comportamiento tridimensional en términos estructurales y litoestratigráficos del mirador hidalgo se infirió mediante los modelos geofísicos resultantes de los estudios de Tomografía Eléctrica Resistiva y Sondeos Electromagnéticos en el Dominio del Tiempo, cuyos perfiles, $\mathrm{A}^{-\mathrm{A}^{\prime}} \mathrm{y}$ $B-B^{\prime}$, respectivamente, se realizaron perpendiculares al corte del mirador, de tal forma que proporcionaran información de cómo se propagaban geométricamente las litologías observadas en el talud. En resumen, los resultados combinados de los caminamientos geológicos y la interpretación de los modelos finales de los perfiles geofísicos permitieron generar una zonificación del corte del mirador hidalgo en términos de un índice de susceptibilidad geológica, definido como la resultante de las sumatorias de los pesos asignados tanto a factores condicionantes como desencadenantes. Finalmente, la zonificación resultante del corte del mirador hidalgo (figura 9), muestra como la Zona I, al sureste del corte, representa el mayor índice en la zona de emplazamiento de la infraestructura del mirador, dado que las litologías prevalecientes en esta zona están dominadas por lutitas parcialmente alteradas o metamorfizadas, extremadamente débiles en cuanto a resistencia al corte. Además de la presencia de intrusivos ígneos altamente fracturados observados sobre el corte, se identificaron derrumbes de este tipo de roca, evidenciando la relativa facilidad con que se desprenden del macizo rocoso sobre el cual descansa el mirador. En esta zona no se presenta un volumen apreciable de calizas masivas que provean estabilidad al edificio geológico. La falla geológica identificada como F1 es una condicionante estructural extrema de cómo el macizo de roca ígnea ha sido afectado por efectos estructurales posteriores a su emplazamiento. Es decir, esta falla induce el colapso del macizo rocoso en forma de flujos de roca o derrubios de hasta $5 \mathrm{~m}$ de diámetro que llegan hasta el primer carril del Camino Real.

En la Zona II, aún y cuando se observa que las terrazas de estabilización están estructuralmente íntegras y disminuye notablemente la presencia de lutitas expuestas, la geofísica revela que es precisamente esta litología la que soporta el macizo rocoso.
La Zona III, en la base del corte, a nivel de calle se aprecia un lente expuesto de lutita orgánica (color negro) con un índice de dureza R0 (extremadamente débil). Esta unidad litoestratigráfica coincide espacialmente con los sondeos geofísicos, los cuales revelan que, al igual que en la Zona II, esta unidad es la que "carga" o soporta el peso de los estratos correspondientes a rocas ígneas y conglomerados terciarios. Se asigna un menor índice de riesgo dado que la geometría, es decir, la altura que alcanza el corte, es menor, reduciéndose por consiguiente el potencial gravitacional de falla.

La Zona IV no presenta daños estructurales y sólo se evidencian de forma expuesta, unidades de rocas calizas e ígneas, subyaciendo un paquete de conglomerados terciarios. Posteriormente, el macizo de roca ígnea se acuña en dirección noroeste, dejando expuestas sólo litologías del tipo de conglomerado terciario y rellenos antropogénicos compuestos por los clastos generados durante las explosiones que abrieron el corte del Camino Real. En el interior de esta zona, la interpretación de la geofísica revela la presencia de lutitas y calizas alternadas con ángulos de echado superiores a los 70 grados. El problema principal observado en esta zona es el de la presencia de rellenos antropogénicos pobremente consolidados, generados durante la remoción de material. Además de la debilidad estructural de las lutitas, las cuales al presentar elevados ángulos de echado, no soportan cargas excesivas aparentemente.

\section{Referencias}

ABEM, 2008, Instrument AB.

Ávila V.M. An Investigation of the Seismic Hazards of the El PasoJuarez Region: The Nature and Extent of the Southern East Flanklin Mountains Fault Zone. Thesis Proposal (Master of Science), Universidad de Texas en El Paso, 2005.

Ayala C.F. Introducción a los riesgos geológicos, en: Riesgos geológicos, Instituto Geológico y Minero de España, Madrid. 1987.

Centro de Información Geográfica (CIG) [en línea]. Disponible en: http://cig.uacj.mx.

Collins E.W., Raney J.A. Geologic Map and Structure Sections of the Sierra de Juarez, Chihuahua, México, US Geological Survey, Miscellaneous Investigations, Map I-2287. 199.

Comisión Nacional del Agua (CNA). Estudio integral de factibilidad en las cuencas Zona I-Centro y Zona II-Anapra en Ciudad Juárez, estado de Chihuahua, Ciudad Juárez, Chihuahua, 2008.

Constable S.C., Parker R.L., Constable C.G. Occam's InversionA Practical Algorithm for Generating Smooth Models From 
Electromagnetic Sounding Data. Geophysics, 52(03):289-300, 1987.

Dahlin T., Zhou B. A Numerical Comparison of 2D Resistivity Imaging with 10 Electrode Arrays, Geophysical Prospecting, 2004, pp. 379-398.

Dahlin T., Linderman J. ERIGRAPH, Software Developed in Cooperation between ABEM, Lund University and Terraohm, 2007.

Dena O.S. Dictamen geológico del proyecto ejecutivo del Dique Parque Sierra de Juárez. Ciudad Juárez, Chihuahua, IMIP, 2009, pp. 159.

Drewes H., Dyer, R. Tertiary and Quaternary Structure and Paleotectonics of the Hueco Basin, Trans-Pecos Texas and Chihuahua, Mexico. Bureau of Economic Geology. 1993.

Flores R., Vangel V.H., Liu X. Geotecnia en ingeniería de presas, primera edición. México, Instituto Mexicano de Tecnología del Agua, 2001, pp. 583. ISBN: 968-7417-91-9.

Geonics Limited. Protem 47D Operating Manual. Ontario, Canada, 1999, pp. 74.

González de Vallejo L., Ferrer M., Ortuño L., Oteo C. Ingeniería geológica. Madrid, España, Prentice Hall, 2002, 744 pp.

Haenggi W. Tectonic History of the Chihuahua through, Mexico and Adjacent USA, Part II: Mesozoic and Cenozoic. Mexican Geological Society Bulletin, 55(1):38-94, 2002.

Haller K.M., Wheeler R.L., Ruckstales K.S. Documentation of Changes in Fault Parameters for the 2002 National Seismic Hazard Maps-Conterminous United States except California. US Geological Survey Open-File Report, 1 02-467. 2002.

IMIP. Instituto Municipal de Investigación y Planeación [en línea]. Disponible en: http://www.imip.org.mx/

ISRM. Suggested Methods for the Quantitative Description of Discontinuities in Rock Masses. Int. J. Rock Mech. Sci. \& Geomch. 1978. Abstr, 15(6, 127-134).

Keaton J.R. Maps of Potential Earthquake Hazards in the Urban Area of El Paso, Texas: Final Report, under Award, U.S. Geological Survey, National Earthquake Hazards Reduction Program, Program Element III, 1993. 14-08-001-G2171.

Keaton J. R., Barnes J. Paleoseismic Evaluation of the East Flanklin Mountains Fault, El Paso, Texas: Final Report, under Award, U.S. Geological Survey, National Earthquake Hazards Reduction Program, Program Element III, 1996. 1434-94-G-2389.

Keller G.R., Morgan P., Seager W.R. Crustal Structure, Gravity Anomalies and Heat Flow in the Southern Rio Grande Rift and their Retion Ship to Extensional Tectonics. 1990.

Laboratorio de Climatología y Calidad del Aire de la Universidad Autónoma de Ciudad Juárez [en línea].Disponible en: http:// www2.uacj.mx/clima/

Loke M.H. Electrical Imaging Surveys for Environmental and Engineering Studies. 2000.

Loke M.H. Tutorial: 2-D and 3-D Electrical Imaging Surveys. 2004.

Loke M.H. Electrical Imaging Surveys for Environmental and Engineering Studies: A Practical Guide to 2-D and 3-D Surveys.
Unpublished Short Training Course Lecture Notes, Geotomo Software, Penang, Malaysia, 2000, 65 pp.

Loke M.H. Tutorial: 2-D and 3-D Electrical Imaging Surveys [en línea] Disponible en: http://www.umt.edu/geosciences/faculty/sheriff/495-subsurface imaging in archaeology/Sources/ Loke_elect_tutorial.pdf, 2004.

Lovejoy E.M. An Interpretation of the Structural Geology of the Franklin Mountains, Texas: New Mexico

Geological Society Guidebook $26^{\text {th }}$ Field Conference, Las Cruces Country, 1976, pp. 261-268.

Machette M.N. Preliminary Assessment of Paleoseismicity at White Sands Missile Range, Southern New Mexico- Evidence for Recency of Faulting, Fault Segmentation, and Repeat Intervals for Major Earthquake in the Region: U.S. Geological Survey Open-File Report 1987, 87-444, pp. 46.

Mendoza M.J., Domínguez L. Noriega I., Guevara E. Monitoreo de laderas con fines de evaluación y alertamiento. México DF, CENAPRED, 2002, pp. 78.

Palacky G.I., Resistivity Characteristics of Geological Targets, en: M. Nabighian, Editor, Electromagnetic Methods in Applied Geophysics-Theory, Society of Exploration Geophysicists, Tulsa, OK, 1987, pp.53-129.

Rascón E., Gómez F.J. Estudio de factibilidad para el control integral de avenidas en las cuencas Zona I

Anapra y Zona II-Centro en Ciudad Juárez, en el estado de Chihuahua, Reporte interno, CNA, 2008, pp. 890.

Raney J.A., Collins E.W. Regional Geologic Setting of the Fort Hancock Study Area, Hudspeth Country, Texas: Bureau of Economic Geology, Final Contract Report Prepared for Texas Low-Level Radioactive Waste Disposal Authority, 1990, pp. 69.

RES2DINV. Geotomo Software, 2003.

RockWorks. Earth Science and GIS Software, 2002.

Shah S.D., Kress W.H., Land L.A. Time-Domain Electromagnetic Soundings to Characterize Water Quality Within a Freshwater/ Saline-Water Transition Zone, Estancia Valley, New Mexico, July 2005-A Reconnaissance Study, Fact Sheet fs 2007-3011.

Sharma P. Environmental and Engineering Geophysics, Cambridge University Press, 1997, pp. 500, ISBN: 0521576326.

Telford W.M., Geldart L.P, Sheriff R.E. Applied Geophysics. 2 ${ }^{\mathrm{a}}$ Edicion, Cambridge University Press, 1995, pp. 500. ISBN: 0-52133938-3.

Tovar R. Stratigraphy and Tectonics of the State of Chihuahua, México, en: Geologic Field Trip thru the State of Chihuahua. 1974.

Webb D.S. Facts of the Geology of the Sierra del Presidio Area, North-Central Chihuahua, en: The Border Region: New Mexico Geological Society $29^{\text {th }}$ Field Conference Guidebook, 1969, pp. 182-185.

Wessel P., Smith,W.H. GMT Version 4.1.4. A Map-Making Tutorial, School of Ocean and Earth Sciences and Technology and NOAA/NESDIS. 


\section{Semblanza de los autores}

Oscar S. Dena-Ornelas. Es doctor en geofísica por parte de la Universidad de Texas en El Paso. Actualmente dirige el Centro de Información Geográfica del Instituto de Ingeniería y Tecnología de la Universidad Autónoma de Ciudad Juárez. Ha realizado investigaciones en las áreas de sísmica de refracción de baja y media profundidad y reflexión sísmica en Marathon Oil Company. Ha realizado estudios de hidrología, geología y geofísica ambiental, así como estudios de riesgo geológico y sísmico.

Griselda Obeso-Cortez. Es ingeniera en física por parte de la Universidad Autónoma de Ciudad Juárez, actualmente se encuentra realizando un estudio de gravimetría en el acuífero de Conejos Medanos y se especializa en sistemas de información geográfica en el Centro de Información Geográfica. Ha realizado investigaciones en el área de geofísica ambiental.

Jesús Leyva. Estudiante del último año de la licenciatura en ingeniería física, actualmente se encuentra realizando su tesis de licenciatura en tomografías eléctricas resistivas y gravimetría.

Miguel Domínguez-Acosta. Es doctor en geología por parte de la Universidad de Texas en El Paso. Actualmente se encuentra adscrito al Departamento de Ingeniería Civil y Ambiental de la Universidad Autónoma de Ciudad Juárez como profesor-investigador de tiempo completo. Ha realizado estudios de geomorfología en ambientes sedimentarios eólicos y lacustres en el desierto chihuahuense al igual que estudio de cartografía hidrogeoquímica en el suroeste de Estados Unidos.

Víctor Hernández-Jacobo. Es maestro en ingeniería, actualmente dirige el Departamento de Ingeniería Civil y Ambiental de la Universidad Autónoma de Ciudad Juárez. Sus áreas de investigación son mecánica de suelos y geotecnia.

Alfredo Granados-Olivas. Es doctor en agronomía por la Universidad Estatal de Nuevo México. Se especializa en sistemas de información geográfica y sistemas de teledetección con sensores remotos.

Servio Tulio de la Cruz-Cháidez. Es doctor en estructuras por la Universidad Politécnica de Cataluña. Actualmente dirige el programa de maestría en ingeniería civil de la Universidad Autónoma de Ciudad Juárez. Se especializa en diseño y evaluación de estructuras. 